\title{
LA REFORMA ESTRUCTURAL DE LA ADMINISTRACIÓN TRIBUTARIA EN EL PERÚ: 1991 - 1997
}

C.P.C. PAULINO BARRAGÁNARQUE

Profesor Asociado de la UNMSM; Profesor Investigudor del Instituto de Investigaciones de la Facullad de Ciencias Administrativas de la UNMSM. Funcionario de la SUNAT. Graduado en La Universidad Nacional de San Agustín de Arequipa; Maestría en la UNMSM; Post Grado en La Escuela Interamericana de Administración Publica . EIAP - Rio de Janeiro - Brasil; Instituo para el Desarrollo Internacional - DSE - Berlín Occidental - Alemania; Centro Interamericano de Administradores Tributarios - CIAT - Panamá.

\section{SITUACIÓN ECONÓMICA DEL PERÚ EN JULIO DE 1990}

La reforma estructural de la administración tributaria peruana, sc inició en el contexto en el cual el Perú atravesaba, la más grave crisis económica de su historia republicana, caracterizada por una hiperinflación que en julio de 1990 alcanzaba el $7,650 \%$ anual, una aguda recesión y distorsión de los precios relativos, una severa caída en el nivel de producción, el desprestigio de sus más altas autoridades así como la existencia de un sanguinario grupo terrorista denominado Sendero Luminoso, que desde 1980 ya había causado más de 20 mil muertes.

Desde el punto de vistá económico, existían serios desequilibrios tanto en la balanza comercial como en las finanzas publicas. En el campo externo el país estaba aislado del Sistema Financiero Internacional, no contaba con reservas internacionales, siendo negativa la disponibilidad de reservas netas; mientras en el campo interno el déficit fiscal alcanzaba el 13\% de PBI y la presión tributaria descendía a niveles cercanos del $4.5 \%$ del PBI, en el primer sernestre de 1990,siendo una de las más bajas en el nivel internacional.

El bajo nivel de la recaudación tributaria, era el resultado del colapso del Sistema Tributario Nacional, tanto del conjunto de tributos existentes compuesto por más de 97 clases de gravámenes e innumerables privilegios fiscales; como de la administración tributaria, mal preparada, mal equipada y además corrupta. Junto con ello el sector informal de la economía en continua expansión, el proceso heperinflacionario, habían contribuido con erosionar las bases sobre las cuales se contribuía con el fisco.

Para hacer frente a esta situación de anarquía y caos financiero que vivía el país, el Gobierno puso en marcha un Programa de Estabilización Económica a partir del 8 de agosto de 1990, el mismo que estuvo orientado, a eliminar de raíz las fuentes de desequilibrio financiero, anulándose los subsidios sustentados en el tipo de cambio preferencial, iniciándose un manejo 
estricto de las finanzas públicas, conducente a la eliminación del financiamiento monetario del déficit fiscal; se unificó el mercado de divisas adoptándose un sistema de flotación cambiaria; se corrigió el atraso de los precios públicos y se establecieron tasas de interés determinadas por el mercado.

El Programa de Estabilización Económica estuvo orientado también a llevar a cabo el proceso de reintegración del Penú a la Comunidad Financiera Internacional y definir un marco adecuado para el crecimiento en el mediano plazo, mediante la realización de una serie de reformas estructurales. También se inicio las reformas del sistema de incentivos a la actividad productiva, tales como las reducciones arancelarias y la eliminacion de las restricciones cuantitativas y a las importaciones dando prioridad al rol del mercado en la formación de precios.

El sustento del Programa, fue el mantenimiento de una estricta disciplina fiscal y monetaria, orientado a la eliminación de déficit fiscal del sector público, mediante el incremento de los ingresos tributarios. Asimismo, el nivel del gasto se definió en función de la disponibilidad de ingresos; cuyo manejo está actualmente bajo la responsabilidad de un Comité de Caja, a la vez que la política de precios públicos buscó evitar atrasos en sus precios relativos, lo que hicieron posible detener el proceso hiperinflacionario de $7,650 \%$ en 1990 a $139.2 \%$ a fines del año de 1991 ,creciendo el PBI en dicho periodo en $2.6 \%$, revertiendo la tendencia negativa mostrada en los años $1,988,1989$ y 1990.

Dentro de este contexto, el Gobierno tomó conciencia del rol fundamental que le tocaba desempeñar a la administración tributaria, comprendiendo, que la única manera de consolidar las reformas estructurales emprendidas en el segundo semestre del año 1990, y que estas además sean sostenibles en el mediano y largo plazo, era imprescindible efectuar una profunda reforma del Sistema Tributario Nacional; la misma que debía iniciarse con la reforma estructural de la Administración Tributaria.
2. LAS BASES ESTRATÉGICAS DE

\section{LA REFORMA ESTRUCTURAL.}

\subsection{DIAGNOSTICO INICIAL}

La Administración Tributaria Peruana, hasta 1988 era un órgano de línea del Ministerio de Economía y Finanzas, MEF, la antigua Dirección General de Contribuciones dependía del Vice-Ministro de Hacienda, al igual que la Dirección General de Aduanas y las direcciones de Presupuesto, Tesoro, y Crédito Público. La gestión de los recursos presupuestales y financieros dependía enteramente del MEF, en la medida que el presupuesto era aprobado y ejecutado en función de las prioridades establecidas por la Oficina General de Administración del mismo.

Esta situaciôn cambio, al otorgarse autonomía formal a la Administración Tributaria, con la dación de la Ley Nro. 24829 del 07.06.88 que cré́ la Superintendencia Nacional de Administración Tributaria - SUNAT - y la Superintendencia Nacional de Aduanas - ADUANAS -, como instituciones publicas descentralizadas del Sector Economía y Finanzas, contando con patrimonio propio y teniendo como ingresos el $2 \%$ de la recaudación de los tributos que administran; sus leyes generales fueron aprobadas mediante los Decretos Legislativo Nros. 501 y 500 de fecha 29.11 .88 respectivamente.

Este cambio fue más bien de forma que de fondo, por cuanto la administración tributaria en el caso de SUNAT continuó funcionando hasta mediados de 1991 en las mismas instalaciones del Ministerio de Economía y Finanzas; los recursos de gestión presupuestal, se seguían administrando inadecuadamente y la corrupción era un fenómeno extendido en toda la administración tributaria.

\subsection{PILARES ESTRATÉGICOS DE LA REFORMA}

En marzo de 1991 se inició el proceso de la 
reforma estructural de la Administración Tributaria. Ella conllevó el paso de la autonomía formal a la autonomfa efectiva, orientado en primer lugar al surgimiento de una institución moderna y eficiente y en segundo lugar al logro de un sistema tributario equitativo y simple, en el cual tributen todos los contribuyentes señalados por la ley como sujetos del impuesto:

En el desarrollo de este objetivo, concurrieron los siguientes elementos estratégicos:

a. Se contó con el apoyo político del más alto nivel que provino del Presidente de la República y del Congreso.

b. Este apoyo fue compartido por la mayoría de partidos políticos representados en el Congreso de la República y por la opinión pública en general, por que el Estado aparecía como capaz de atacar con eficiencia los problemas más agudos (reducción de la inflación, lucha contra el terrorismo) Por primera vez después de muchos años, las prestaciones estatales parecían tener cierta relación con los impuestos, estos ya no daban la impresión de ser dilapidados por un Estado corrupto e ineficiente.

c. La conducción de la reforma, desde el diagnostico inicial, fue liderada por profesionales calificados que provenían del Banco Central de Reserva - BCR y de técnicos y profesionales expertos de la propia administración tributaria (Ex - Dirección General de Contribuciones)a los que se sumaron los téenicos enviados por los Organismos Internacionales, tales como: el Fondo Monetario Internacional - FMI; Banco Interamericano de Desarrollo - BID; y el Centro Interamericano de Administradores Tributarios - CIAT.

d. El equipo nacional conjuntamente con la Cooperación Técnica Internacional, diseñaron un plan de corto y mediano plazo definiendo las orientaciones básicas y la secuencia de la aplicación de las medidas más importantes. e. Para llevar a cabo la reforma, no solo se dotó a la administración tributaria de los recursos financieros necesarios, sino que también se le dio la capacidad de disponer de ellos oportunamente. En ese sentido, la autonomía en el manejo de los recursos financieros fue un factor muy importante en el éxito alcanzado.

\subsection{MEDIDAS ADOPTADAS EN EL ÁMBITO LABORAL}

a. El equipo que lideró la reforma estructural, como estrategia de trabajo optó por priorizar la evaluación y selección de personal existente a marzo de 1991, por cuanto se llegó a la conclusión, de que no era posible lograr resultados permanentes y sostenidos, sino se contaba con los recursos humanos adecuadamente seleccionados y capacitados

b. Para ello fue indispensable efectuar una profunda reforma laboral sustentada en el Decreto Legislativo Nro. 639 que declaraba en reorganización la Administración Tributaria y dejaba en suspenso la estabilidad laboral de sus trabajadores estableciendo un programa de retiro voluntario con incentivos económicos o acogerse un conjunto de pruebas y exámenes de cuyo resultado dependía su permanencia en la institución.

c. La racionalización de personal aplicada, cesó el vinculo laboral de más de 2,000 trabajadores del personal existente. La reducción más significativa fue en las áreas de fiscalización, en donde se registraba los más altos índices de corrupción.

d. Concluida la racionalización de personal, el Poder Ejecutivo aprob6 el Decreto legislativo Nro.673 del 23/09/91 mediante el cual la Superintendencia Nacional de Administración Tributaria quedaba sujeta al régimen laboral del sector privado, Ley 4916, permitiendo de esta manera pagar sueldos competitivos simila- 
res a los del sector privado.

e. La estrategia para incorporar a nuevo personal se baso, en la selección de jóvenes profesionales no mayores de 27 años pertenecientes a los tercios superiores de estudios universitarios de las carreras de: Contabilidad, Economía, Derecho, Informática y Administración.

\section{SISTEMAS BÁSICOS DESARROLLADOS}

El éxito de la reforma estructural de la Administración Tributariamente entre otras variables del plan estratégico diseñado, ha sido fundarnentalmente, por el constante esfuerzo efectuado por ampliar la base tributaria y el número de contribuyentes; las mismas que están sustentadas en los siguientes sistemas desarrollados.

\subsection{SISTEMA DE REGISTRO ÚNICO DE CONTRIBUYENTES}

El Registro Único de Contribuyentes - RUC, sustituyo a la antigua Libreta Tributaria, del cual se habían emitido más de 6'000,000; sin embargo tal número no correspondía al volumen de contribuyentes que cumplían con sus obligaciones formales y sustanciales, dándose el caso que muchos contribuyentes poseían más de una, lo que no permitía conocer con certeza la dimensión del universo de contribuyentes sujetos a las obligaciones tributarias, y en la práctica se había convertido en un documento más de identidad personal, junto a la Libreta Electoral y la Libreta Militar, que obtienen los ciudadanos de nuestro país al cumplir los 18 años de edad.

El RUC, es uno de los principales sistemas puestos en marcha por la Administración Tributaria en el proceso de la reforma. En este sistema se registran únicamente los contribuyentes de interés para el fisco, aquellos que verdaderamente son alcanzados por las normas tributarias como sujetos de la obli- gación tributaria; incluso aquellas personas cuyos tributos le son totalmente retenidos por terceros no están obligados a tener RUC (como es el caso de los contribuyentes que perciben rentas de primera categoría: los propietarios que alquilan viviendas; $o$ los que perciben rentas de quinta categoría, etc.). El proceso de empadronamiento al RUC, desde su implementación se ha ido perfeccionando hasta llegar a la actual etapa en la que el registro de los contribuyentes y la entrega del número de RUC es efectuado, en tiempo real, en el instante; además, debe ser renovado periódicamente, para evitar que con el paso de los años se desactualice, como ocurrió con la Libreta Tributaria.

\subsection{SISTEMA DE CONTROL DE LA RE- CAUDACIÓN TRIBUTARIA}

El Sistema de Control de la Recaudación Tributaria se puso en marcha desde julio de 1993, sustituyendo el antiguo sistema de recaudación que estaba bajo la responsabilidad del Banco de la Nación, que en virtud de la Ley Nro. 15600 era el único agente financiero del Estado Peruano.

Con el nuevo sistema, se descentralizó la recepción de los pagos directos de tributos, incorporando a esta tarea a los 12 bancos más importantes del Sistema Financiero Nacional, que en conjunto forman una RED BANCARIA que cubren con más de $90 \%$ de las oficinas bancarias a escala nacional.

Mediante el sistema de la recaudación bancaria se controla las declaraciones y pagos de más de 930,000 contribuyentes, de los cuales más del $80 \%$ realizan declaraciones y pagos en más de 1,200 agencias bancarias. Mediante convenios celebrados con los bancos privados y estatales, estos se encargan de recibir las declaraciones y pagos de los contribuyentes, y luego mediante un proceso posterior se encargan de digitar la información de las declaraciones recepcionadas, realizar los abonos correspondientes a la cuenta corriente del Tesoro Publico y realizar las conciliaciones correspondientes en- 
tre los montos declarados, pagados y abonados.

En ese sentido la RED BANCARIA, no solo controla los pagos de las obligaciones tributarias de los contribuyentes, sino que primordialmente, captura la información a través de medios magnéticos para los efectos de conciliación de la recaudación y del control estadístico y contable de la gestión tributaria.

El proceso de la conciliación bancaria es de gran precisión, realizándose documento por documento, lo que permite tener una relacíńn completa de los contribuyentes que efectuaron pagos asociada a la Nota de Abono Bancario.

Es importante destacar que el software de digitación de la información, a sí como las características de hardware utilizados, han sido elaborados por la propia Administración Tributaria. siendo el sistema lo suficientemente versátil para prevenir cualquier modificación que se origine en los cambios legislativos, convirtiéndose así en uno de los sistemas de control de la recaudación tributaria, lideres en América Latina.

\section{ELSISTEMA DE COMPROBANTES DE PAGO}

Mediante el Decreto Ley Nro. 25632 de julio de 1992, se estableció el nuevo régimen de comprobantes constituidos por las facturas, boletas de venta, recibos por honorarios profesionales, tickets, boletos de máquinas registradoras, liquidaciones de compra, etc. Se definió además que el emisor, debía efectuar una declaración previa a su impresión y/o al uso de sistemas computarizados, a efectos de llevar un control especial de las solicitudes de impresión y de las series autorizadas a través de un software especial. También se estableció que las imprentas tienen la obligación de registrarse en la SUNAT, y realizar sus trabajos de impresión solo a los contribuyentes que les presenten la declaración previa.
Mediante este sisterna más de 6,500 imprentas, deben entregar información trimestral a la Administración tributaria sobre los trabajos de impresión realizados, siendo una importante herramienta para combatir la evasión tributaria.

Es importante destacar que para tener derecho a utilizar las facturas como sustento del crédito fiscal o de gastos en los registros y balances contables, las facturas deben encontrarse en la base de datos de la Administración Tributaria.

\subsection{EL SISTEMA DE CONTROL DE PRIN- CIPALES CONTRIBUYENTES NA- CIONALES}

La estrategia de la recaudación tributaria diseñada por la Administración Tributaria se basó en un tratamiento diferenciado entre los denominados Principales Contribuyentes, los mismos que por su importancia en términos de recaudación cumplen con efectuar sus declaraciones y pagos en las propias oficinas de la Administración Tributaria a nivel nacional, y los Medianos y Pequeños Contribuyentes, cumplen con sus declaraciones y pagos en cualquiera de las oficinas de la red bancaria; sin embargo esto es lo único que los diferencia de los Principales Contribuyentes, puesto que el software desarrollado para capturar los datos de las declaraciones, realizar los abonos del dinero a la cuenta corriente del Tesoro Público, realizar las conciliaciones bancarias, son las mismas que se utilizan para el Sistema de Principales Contribuyentes Nacionales. Mediante el Sistema de Principales Contribuyentes Nacionales, se controlan las declaraciones y pagos de más de 17,000 contribuyentes en todas las oficinas de la SUNAT a nivel nacional. La recaudación de estos contribuyentes representan más del $80 \%$ de la recaudación total. En estas oficinas los datos de las declaraciones ingresan "on line", permitiendo de esta 
manera detectar en la misma ventanilla de pago cualquier error en las declaraciones y actuar sobre los contribuyentes omisos a la presentación de las declaraciones o pagos dentro de las 24 horas de ocurridos los vencimientos de las obligaciones tributarias.

\section{RESULTADOS OBTENIDOS}

Después de 7 años de haber puesto en marcha la más profunda reforma estructural de la Administración tributaria efectuada en nuestro país, y como resultado de las medidas adoptadas y los sistemas implementados, se han obtenido resultados muy halagadores y trascendentales, entre los que podemos destacar, los siguientes:

4.1. Se ha logrado reconstruir y simplificar el Sistema Tributario Nacional, el cual a junio de 1990 había colapsado, tanto política como administrativamente. Dos han sido los elementos que han caracterizado la reforma impositiva: la neutralidad, a través del cual se han reducido drásticamente las principales distorsiones de nuestra economía, y el aumento de Ia eficiencia, tanto de la política como de la administración tributaria, que se refleja en la simplificación del sistema tributario, la modernización de su administración y la introducción con fuerza de la tecnología de punta (nuevos sistemas informáticos) al control de la recaudación y al cumplimiento de las obligaciones tributarias.

4.2. La presión tributaria con respecto al PBI. se incremento de $4.5 \%$ en junio de 1990 a $14.3 \%$ y 14.1 en 1996 y 1997 respectivamente (ver cuadro Nro. 1). El equilibrio fiscal ha sido un elemento clave en la derrota de la inflación, el mismo, que actualmente no pasa de dos dígitos anuales.

\section{Cuadro $N^{*} 1$ \\ EVOLUCIÓN DE LA PRESIÓN TRIBUTARIA}

(Porcentaje de PBI)

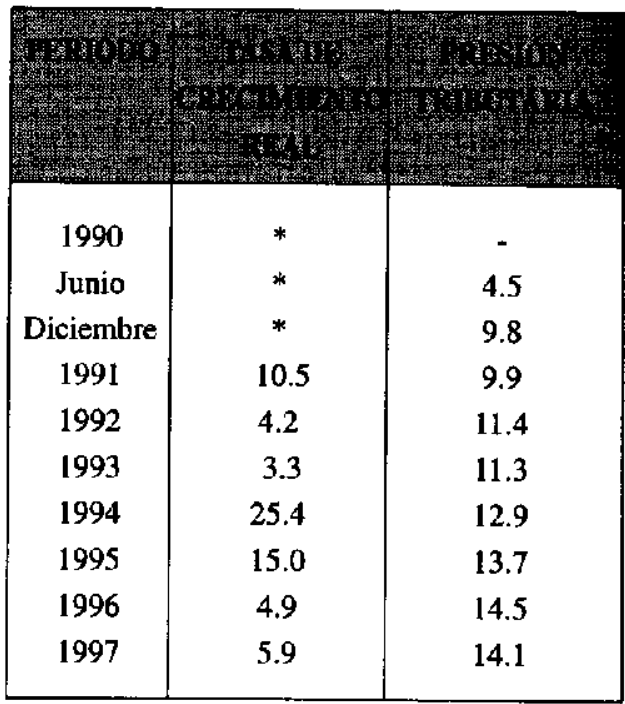

Fuente: SUNAT

4.3 Se ha logrado un avance muy importante en la simplificación del Sistema Tributario $\mathrm{Na}$ cional, en la actualidad, la recaudación se basa principalmente en la aplicación de dos impuestos: el Impuesto General a las Ventas y el impuesto a la Renta. En conjunto ambos impuestos representan el $69 \%$ del total recaudado, lo que brinda una gran estabilidad a la recaudación y la hace menos vulnerable, a cambios en los precios relativos o variaciones sectoriales internas y externas. A junio de 1990 el sistema tributario era altamente dependiente del precio de los combustibles, del sector minero o del tipo de cambio; tal como se puede observar en el siguiente cuadro estadístico: (ver página siguiente).

4.4. La base tributaria de contribuyentes que declaran y pagan sus obligaciones tributarias se ha ampliado notablemente; además se hacen importantes esfuerzos por incorporar al sector informal a traves de regímenes tributarios especiales como 


\begin{tabular}{|c|c|c|c|c|c|c|c|c|}
\hline I.G.V.' I & 13.6 & 24.6 & 30.6 & 0.0 & 43.7 & 44.5 & 41.9 & 430 \\
\hline A LA RENTA & 6.1 & 8.1 & 13.3 & 16.3 & 18.9 & 20.4 & 25.0 & 24.9 \\
\hline ALA IMPORTACION & 11.4 & 11.3 & 10.1 & 11.6 & 11.4 & 11.9 & 11.0 & 9.9 \\
\hline ISC COMBUSTIBLES & 24.8 & 22.3 & 17.0 & 11.5 & 9.6 & 8.7 & 7.9 & 8.3 \\
\hline ISC OTROS & 12.3 & 14.9 & 13.5 & 7.2 & 7.3 & 6.1 & 5.8 & 6.2 \\
\hline FONAVI & 3.2 & 4.1 & 5.1 & 6.1 & 6.4 & 6.3 & 6.9 & 6.2 \\
\hline OTROS & 28.6 & 14.7 & 10.4 & 7.3 & 2.7 & 2.1 & 1.6 & 2.3 \\
\hline
\end{tabular}

1 Incluye Impuesto de Promoción Municipal

Fuente: SUNAT

con son: el Régimen Único Simplificado y el Régimen Especial de Renta. El número de contribuyentes que cumplen con declarar (presenta formularios de declaración/pago) oportunamente actualmente son en un promedio de 680,000 , de los cuales pagan un promedio 450,000

4.5. Otra de las características de proceso de la ampliación de la base tributaria lo constituye el incremento del numero de principales contribuyentes bajo control, la gran mayoría son personas jurídicas: en diciembre de 1990 eran alrededor de 670 contribuyentes, en la actualidad se administran más de 17,000 contribuyentes, mediante un sistema informático especialmente diseñado llamado control "on line", que permite la liquidación inmediata de tributos, la emisión de acotaciones y la obtención de los reportes diarios de recaudación.

\subsection{La Recaudación por ente administrador de} los tributos que constituyen Ingresos del Gobierno Central - Tesoro Público, es el siguiente:
Cuadro $N^{\circ} 3$

INGRESOS POR ENTE RECAUDADOR

En millones de soles

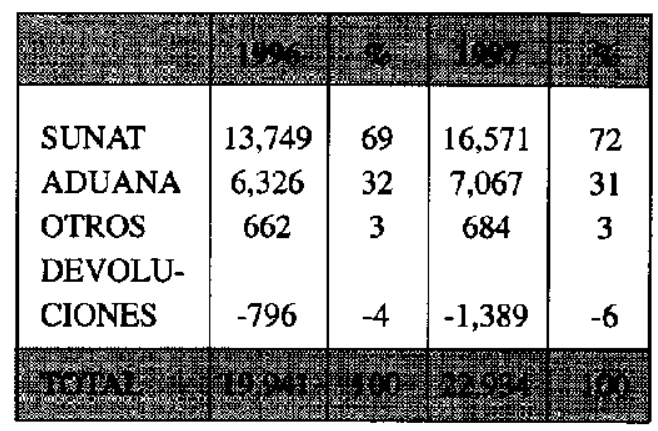

Fuente: SUNAT

4.7. Se ha logrado avances muy significativos en el desarrollo de la conciencia tributaria, el mismo que es complementado a través de la educación en los colegios con programas de educación cívica; desarrollando de programas de orientación y atención a los contribuyentes en zonas estratégicas de Lima Metropolitana y en todas las Intendencias Regionales y oficinas de la SUNAT a nivel nacional. 
4.8. La Superintendencia Nacional de Administración Tributaria, SUNAT, después de 7 años de haberse puesto en marcha la más profunda reforma estructural, se ha constituido en una de las instituciones más sólidas y prestigiosas del país, integrada por personal altamente calificado, identificado con los objetivos institucionales; con un eleva. do sentido de la ética, la moral y la honestidad. En el contexto internacional esta considerada como una de las administraciones tributarias más desartolladas de América Latina, junto a sus similares de Chile y Argentina.

\section{BIBLIOGRAFÍA}

1. Econ. MANUEL ESTELA BENAVIDES, Superintendente Nacional de administración Tributaria: periodo 1990, 1992. Reforma del Sistema y la Administración Tributaria. Documento de Trabajo publicado por el Instituto de Administración Tributaria - IAT.

2. Mg. Econ. LUIS ALBERTO ARIAS MINAYA Superintendente Nacional Adjunto de la Superintendencia Nacional de Administración Tributaria: periodo 1990, 1994. El Control de Gestión de la Administración Tributaria en el Perú. Ponencia presentada en la XXVIII Asamblea del Centro Interamericano de Administradores Tributarios CIAT, realizada en Quito - Ecuador, en Junio de 1994.

3. PhD. FRANCISCO D U R A N D ARP-NISSEN. Revoluciones Tributarias y relación Estado - Sociedad en Bolivia,
Argentina y Perú, Documento presentado en el XVIII Congreso Intemacional de la Asociación Americana de Estudios Latinoamericanos celebrado en Atlanta EE.UU. en marzo de 1994,traducido por el IAT. y publicado en Documentos de Trabajo.

4. Econ. LUIS ALBERTO ARIAS MINAYA. El Fortalecimiento Institucional de la Administración Tributaria Peruana, Documento de Trabajo presentado por el Instituto de Administración Tributaria

5. Banco Central de Reserva del Perú - BCR. Memorias anuales del - 1990 y 1991

6. SUNAT . Nota Tributaria. Publicación mensual.

7. Oficina de Informática y Estadística del MEF. Compendio de los Indicadores Macroeconómicos. Publicación mensual. 